\title{
Beyond Standard Model Searches at the Tevatron
}

Gérard Sajot

On behalf of the CDF and D0 collaborations

LPSC, Université Joseph Fourier Grenoble 1, CNRSIN2P3

53 rue des Martyrs 38026 Cedex Grenoble FRANCE

\section{Introduction}

CDF and D0 have been working at the energy frontier at the Tevatron for many years and have gained experience in the detectors responses to all particle types. They can look at a large number of different final states searching for deviations from Standard Model (SM) expectations and interpret them in terms of new models. The results presented here are a selection of recent searches conducted by the two collaborations using up to $6.3 \mathrm{fb}^{-1}$ of data. They are quoted as they were presented at the time of PLHC2011 regardless of any later updates. Further details on physics results can be obtained from the web pages of the two collaborations [1].

\section{Same sign dileptons}

A wide variety of models for new physics predict events with two like-sign leptons, a signature which has very low backgrounds from SM processes. CDF has studied an inclusive like-sign dilepton sample [2] using $6.1 \mathrm{fb}^{-1}$ of data and interpreted the results in term of :

- Supersymmetry (SUSY)

Analysis has been done in the framework of a practical SUSY model by choosing specific production and decay modes, and including in the model the minimal necessary particle content [3]. The parameters of the model are the masses of the sparticles. The results are expressed as limits on the cross section times branching ratio as a function of the squark and gluino masses.

- Like sign top quark pair production

Flavour-changing neutral processes involving top quarks offer a window to new physics because they are highly suppressed in the SM. CDF has presented the first search for like-sign top pair production [4]. Such pairs can be produced at the Tevatron via the exchange of a heavy $s$-channel vector boson, or a $t$-channel one. In the former case the new particle can be a colour-triplet or sextet with charge $4 / 3$, while in the latter it can be a colour singlet $Z^{\prime}$ or octet $g^{\prime}$, both with zero charge. The analysis is done in the hypothesis of heavy boson masses, so these processes can be described by a gauge-invariant effective four-fermion interaction. Limits can eventually be interpreted in the context of various SM extensions. The effective Lagrangian of the process is expressed in terms of dimensionless constants $C_{L L}, C_{R R}, C_{L R}$ ( $L$ and $R$ are the chiralities of the quarks) and $\Lambda$, the scale of the new physics. All data distributions are in agreement with the expected background contributions. Limits are obtained on the production cross section times dilepton branching ratio according to the chiralities.

- $H^{++/--}$search

The CDF collaboration used a generic search for $H^{++/--}[5]$ in which the charged Higgs may be member of a singlet, doublet or triplet [6]. The production process is assumed to be via $q \bar{q} \rightarrow H^{++/--}$followed by the decay of the doubly charged Higgs to charged leptons: $e e, e \mu$, $\mu \mu$. No excess of events is seen in data compared to what is expected from the background. Limits on the mass of the doubly charged Higgs are obtained (Fig. 1). 

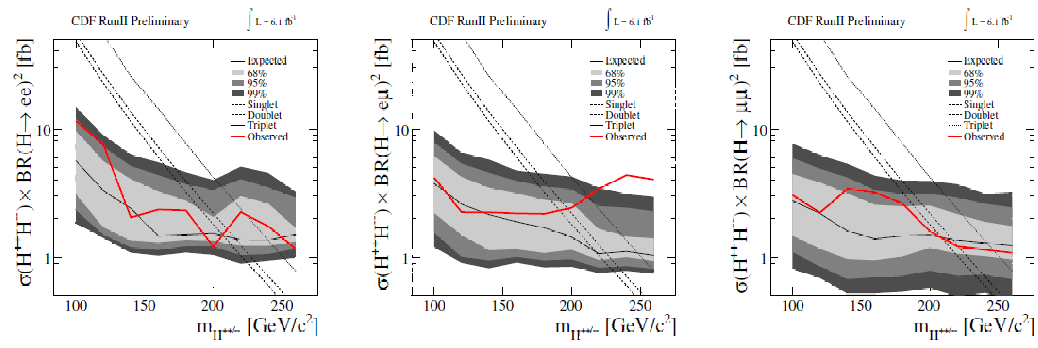

Figure 1: CDF $H^{++/--}$search : Observed and expected limits compared to the theoretical predictions for ee(right), $\mathrm{e} \mu$ (middle) and $\mu \mu$ (right).
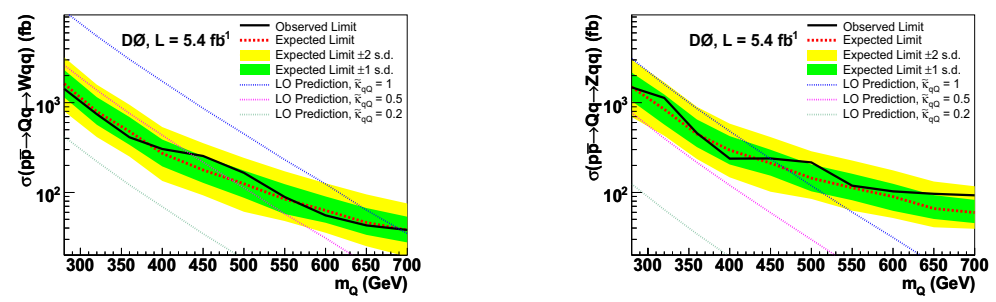

Figure 2: Excluded production cross section for a vector-like quark Q decaying to $W q$ (left) or to $Z q$ (right) compared to LO predictions for various couplings (see [7] for details).

\section{Single vector-like quarks}

Among the theories that have been proposed to describe phenomena at the $\mathrm{TeV}$ scale, some of them predict the existence of massive vector-like quarks (VQ). Their left and right components transform the same way in SU(3) X SU(2) X U(1). They can be singly produced via the electroweak interaction and may decay into a $W$ or $Z$ boson and a SM quark. D0 [7] has separated the search in two independent channels : lepton, missing transverse energy, and a jet (decay into $W q$ ) and dilepton +jet (decay into $Z q$ ). The absence of any statistically significant excess in data above the expected background allows the derivation of cross section limits. They are compared to VQ production in two scenarios : VQ not coupled to down-quarks and decaying exclusively to $W q$ are excluded for masses below $693 \mathrm{GeV}$ (551 GeV if decaying to $Z q$ ) (Fig. 2). In the scenario of no-coupling to up-quarks, the mass limits are $403 \mathrm{GeV}(W q)$ and $430 \mathrm{GeV}(Z q)$.

\section{$4 W+2$ jets}

Measurements of associated production of a $W$-boson and jets are fundamental to probe the SM and are an essential starting point for searches for physics beyond the SM. CDF has measured the production cross section of a $\mathrm{W}$ in association with either a $W$ or a $Z$ boson from a selection of a 
lepton (electron or muon) plus jets in a data sample of $4.3 \mathrm{fb}^{-1}$ [8]. The best fit to the observed dijet mass distribution using all known components of the background shows an excess of data in the $120-160 \mathrm{GeV}$ mass range corresponding to a 3.2 standard deviations (Fig. 3). Recently CDF has analyzed a larger sample $\left(7.3 \mathrm{fb}^{-1}\right)$, made additional tests and obtained a larger disagreement of 4.1 standard deviations [9]. The D0 collaboration does not confirm such an excess in a data sample of $4.3 \mathrm{fb}^{-1} \quad[10]$.

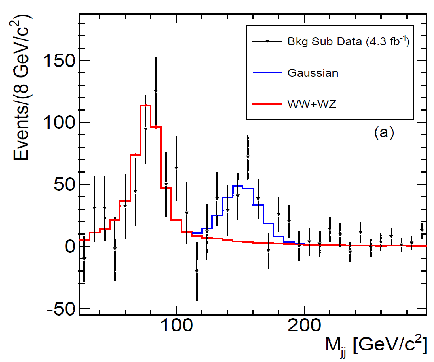

Figure 3: CDF dijet invariant mass distribution for the $W+2$ jets selection where the $W$ decays leptonicaly. The distribution is obtained by adding electron and muon selected events. See $[8]$ for details.

\section{$5 \quad$ Charge asymmetry in like sign $\mu \mu$ events}

The violation of the CP symmetry is a necessary condition for baryogenesis. However, the present observed level of CP violation in $K^{0}$ and $B_{d}^{0}$ and the small expected one from $B_{s}^{0}$ are not sufficient to accommodate this asymmetry, suggesting the presence of additional sources beyond the SM. D0 has measured the like-sign dimuon charge asymmetry in a data sample corresponding to an integrated luminosity of $6.1 \mathrm{fb}^{-1}$ [11]. The periodic reversal of the D0 solenoid and toroid polarities results in a cancellation of most detector-related asymmetries. Two asymmetries are measured. The first one is defined as $A=\frac{N^{++}-N^{--}}{N^{++}+N^{--}}$where $N^{++}$and $N^{--}$represent the number of events in which the two muons of highest transverse $P_{T}$ have the same positive or negative charges. After kinematic selections and background removal, the residual asymmetry is assume to arise solely from the mixing of $B_{q}^{0}$ that then decay semileptonically. The corrected asymmetry is defined as $A_{s l}^{b}$. The second asymmetry is obtained from the measurement of the charge asymmetry in semileptonic decays of b-hadrons : $a=\frac{n^{+}-n^{-}}{n^{+}+n^{-}}$, where $n^{+}$and $n^{-}$correspond to the number of positively and negatively charged muons. The two asymmetries have different contributions from background and detector effects. So $a$ is used to constraint the background contribution to $A$, thereby achieving a further reduction of the total uncertainty on $A_{s l}^{b}$. The final result is $A_{s l}^{b}=-0.00957 \pm 0.00251($ stat $) \pm 0.00146$ (syst). It differs from the SM prediction of $A_{s l}^{b}(S M)=(-2.3+05-0.6) 10^{-4}$ by 3.2 standard deviations. Figure 4 presents the measurement of $A_{s l}^{b}$ in the $a_{s l}^{d}-a_{s l}^{s}$ plane together with direct measurements of $a_{s l}^{d}$ from the B factories [12] and an independent D0 measurement of $a_{s l}^{s}$ in $B_{s}^{0} \rightarrow D_{s} \mu X$ [13].

\section{Conclusions}

The CDF and D0 experiments have accumulated more than $10 \mathrm{fb}^{-1}$ of high quality data and have already set limits on BSM physics beyond what one could have expected. While the LHC has surpassed the reach of the Tevatron in certain areas, CDF and D0 still have, and will continue to have, a competitive BSM physics program, particularly when studying complex final states. 


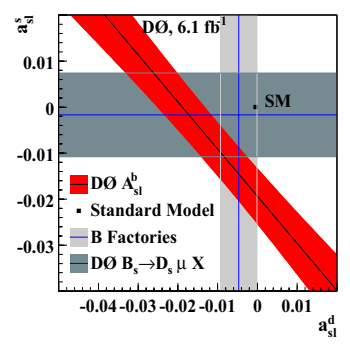

Figure 4: Comparison of $A_{s l}^{b}$ in data with the SM prediction for $a_{s l}^{d}$ and $a_{s l}^{s}$. and other measurements [12] [13]. The bands correspond to $\pm 1 \sigma$ uncertainties.

\section{Acknowledgments}

The author would like to thank the CDF Exotics and D0 New Phenomena groups for providing the material for this talk, and Oscar Gonzalez Lopez, Michael Eads and Michel Jaffré for their advices during the preparation of this talk.

\section{References}

[1] CDF http://www-cdf.fnal.gov/physics/physics.html

D0 http://www-d0.fnal.gov/run2Physics/WWW/results.htm

[2] CDF Collaboration, Public Note CDF 10464.

[3] CDF Collaboration, Public Note CDF 10465.

[4] CDF Collaboration, Public Note CDF 10466.

[5] CDF Collaboration, Public Note CDF 10509.

[6] V. Rentala, W. Shephard and S. Su, arxiv:1105.1379.

[7] V.M. Abazov et al., (D0 Collaboration) PRL 106, 081801 (2011).

[8] T. Aaltonen et al., The CDF Collaboration, Phys. Rev. Lett. 106, 171801 (2011).

[9] Giovanni Punzi, 23th Rencontres de Blois 30 May 2011 and CDF Exotics web pages.

[10] Talk by Aran Garcia-Bellido, this conference.

[11] Phys. Rev. Lett. 105, 081801 (2010).

[12] B. Aubert et al. (Babar Collaboration) Phys. Rev. Lett. 96, 251802 (2006); B. Aubert et al. (Babar Collaboration) arXiv:hep-ex/0607091 (2006).

E. Nakano et al. (Belle Collaboration), Phys. Rev. D 73, 112002 (2006).

E. Barberio et al. (HFAG), arXiv:0808.1297 [hep-ex] (2008).

[13] V.M. Abazov et al. (D0 Collaboration) Phys. Rev. D 82, 012003 (2010). 